\title{
Changing Behaviour to Save Energy: ICT-Based Surveillance for a Low-Carbon Economy in the Seventh Framework Programme
}

\author{
Baki Cakici \\ Swedish Institute of Computer Science (SICS) \\ Stockholm, Sweden \\ Email: cakici@sics.se
}

\author{
Markus Bylund \\ Swedish Institute of Computer Science (SICS) \\ Stockholm, Sweden \\ Email: bylund@sics.se
}

\begin{abstract}
In research and development of information and communication technologies for sustainability, there is a strong belief that human behaviour can be monitored at the individual level to generate different signals, and that these signals can be used to influence individuals to behave differently. We analyse Seventh Framework Programme policy documents published by the European Commission, and descriptions of research projects granted funding from it, to highlight the uncritical development and application of surveillance technologies to change human behaviour. We argue that EU-financed projects dealing with sustainability and information and communication technology use models of social change that have been widely criticised as unlikely to lead to substantial changes in resource consumption. Additionally, we show that these texts discuss only the potential positive effects of technological surveillance, but neither acknowledge nor require the handling of the potential negative effects of surveillance.
\end{abstract}

Index Terms-surveillance, sustainability, behaviour, policy, design, FP7.

\section{INTRODUCTION}

The assumption that energy consumption behaviours can be altered by prompts from computer-supported systems is common in research and development of information and communication technologies (ICTs) for sustainability. Most often, it is assumed that behaviour can be monitored at the individual level to generate different signals, and that these signals can be used to influence individuals to behave differently.

Shove [1] identifies this assumption as belonging to a dominant paradigm of understanding social change as a combination of attitudes, behaviour, and choices within environmental policy. In earlier work, she states that "policies designed to promote sustainable consumption are generally founded upon an extraordinarily narrow understanding of human behaviour" [2, p.111] and argues that this model overemphasises the influence of individual behaviour and individual choice while ignoring many other factors involved in resource consumption. In a similar direction, Dourish [3] details the limitations of systems that frame problems of sustainability as issues of personal choice, and argues for a broadening of the theoretical framework to allow systems to be developed for scales other than the individual.
Focusing on systems that aim to persuade their users to behave differently, Brynjarsdóttir et al. [4] state that such systems are based on a narrow vision of sustainability as resource optimisation, and argue that many such systems tend to provide technological solutions to social problems. These systems reflect specific definitions of sustainability chosen by the designers, and "their framing of sustainability as optimization of a simple metric places technologies incorrectly as objective arbiters over more complex issues of sustainability" [4, p.947].

Other studies within human-computer interaction highlight different targets for intervention aside from individual choices. For example, researchers emphasise the importance of studying everyday interactions [5], [6], and recommend designing systems to actively promote trust among users [7]. Additionally, Dillahunt et al. [8] show that research on sustainability technologies tends to focus on affluent households which make up a narrow demographic, and neglects marginalised groups such as low-income households. The authors also demonstrate that these households engage in energy saving behaviours even when there are no financial incentives.

To these critiques we add an analysis from the perspective of surveillance studies. As DiSalvo, Sengers, and Brynjarsdóttir [9] point out, it is important to engage with questions about "who gets to decide what change should happen and how, whose needs are met and whose values matter in the end" [9, p.1981] when designing ICTs for sustainability. We argue that insights from the field of surveillance studies are well-suited to considering such questions. Ball, Lyon, and Haggerty [10] describe the field as follows:

The contribution of surveillance studies is to foreground empirically, theoretically and ethically the nature, impact, and effects of a fundamental socialordering process. This process comprises the collection, usually (but not always) followed by analysis and application of information within a given domain of social, environmental, economic, or political governance. [10, p.1]

Surveillance systems are used to categorise and classify, and questions about who is included in which categories, and who 
remains excluded follow from their use. Lyon [11] states that surveillance depends on "modes of categorizing populations and treating people differently according to socio-economic status, ethniciy, gender, region, age, and so on" [11, p.177]. These categorisations may benefit their subjects unequally, and as Gandy [12] argues, the differential treatment may become discriminatory.

Surveillance systems and the classifications that they rely on are not neutral. The key point is that there are moral and political consequences of classification [13, p.324]. Classifications that divide information into different categories are affected by the contexts in which these systems are designed in, and they reflect the worldviews of their designers. Cakici [14] discusses issues of classification, and the judgements that result from their use, in the design of ICT-based surveillance systems for smart homes.

In this paper, we are primarily concerned with the uncritical development and application of surveillance technologies. We describe how reliance on the attitude-behaviour-choice model and the use of ICT-based surveillance appear in the Seventh Framework Programme (FP7) policy documents published by the European Commission. With a total budget of 32.4 billion Euros [15], FP7 is highly influential in steering research within European research institutions, and its prominence makes it a good starting point for a critique of ICT-based surveillance. We focus on Challenge 6, ICT for a low carbon economy, within the ICT theme of the Co-operation sub-programme. Based on an empirical investigation of FP7 policy documents and project descriptions that have been financed within the challenge, we argue that EU-financed projects dealing with sustainability and ICT use models of social change that have been widely criticised as unlikely to lead to substantial changes in resource consumption. Additionally, we show that these texts discuss only the potential positive effects of technological surveillance, but neither acknowledge nor require the handling of the potential negative effects of surveillance within everyday interactions.

\section{Methodology}

FP7 collects all research-related European Union (EU) initiatives [15]. It is composed of four sub-programmes: Cooperation, People, Capacities and Ideas. Co-operation is defined as the core programme of FP7. It aims to support collaborative research within and beyond the EU, and it is divided into ten themes: health; food, agriculture and fisheries and biotechnology; information and communication technologies; nanosciences, nanotechnologies, materials and new production technologies; energy; environment; transport; socio-economic sciences and humanities; space; and security.

Our analysis covers the ICT theme of the Co-operation sub-programme within FP7. Each call within Co-operation is associated with a set of documents describing both the content of the call and the application procedures. The primary policy reference in our analysis is the Cooperation Work Programme: Theme 3 - ICT document, which has been with revised six times over the course of FP7, initially appearing in 2007, and with the most recent revision in 2012. Three of these revisions (in 2007, 2009, and 2011) introduce major changes to the challenges and objectives listed in the document, such as renamed or merged items, whereas the intermediate years (2008, 2010, and 2012) bring only minor modifications. The ICT calls are broken into smaller categories called Challenges. Each challenge is further divided into sub-categories called Objectives, and these use a consistent numbering scheme throughout FP7. For example, the label ICT-2009.1.4 refers to the fourth objective within the first challenge of the 2009 version of the policy document.

In our analysis, we used the three major versions of the policy document, and identified the objectives that are of interest for each challenge. To choose the relevant objectives, we searched primarily for descriptions of surveillance targeting humans, that is, systems which monitor, sort, and classify properties of humans and human behaviour directly or indirectly. We conducted the analysis by marking all references to the hypothesis that individual behaviour can be shaped through a combination of surveillance and feedback mechanisms in the collected documents. From this material, we compiled a collection of expected societal impacts of these types of technologies, in particular those that referenced optimisation and rationalisation. We also compiled references to different surveillance activities and expected behavioural changes. From the three versions of the policy document, we selected 15 objectives for further investigation using the same criteria. These objectives were distributed over four challenges which are modified and moved during the three revisions. Using the search engine of the Community Research and Development Information Service (CORDIS) [16] we identified 173 projects which were financed under the objectives we selected.

To limit our material for this paper, we chose to focus on a single challenge originally titled Challenge 6: ICT for Mobility, Environmental Sustainability and Energy Efficiency, and renamed in 2011 to Challenge 6: ICT for a low carbon economy. Out of the 15 objectives we had originally picked, five fell under this challenge, and they contained a total of 55 projects. In terms of the number of financed projects, Challenge 5: ICT for Health, Ageing Well, Inclusion and Governance included a comparable amount of projects. Given the two options, we chose to proceed with Challenge 6 in our analysis primarily due to our greater familiarity with ICT within the domain of sustainability.

We retrieved the project descriptions for the 55 projects from CORDIS. Our analysis is based on these project descriptions as well as the policy documents described earlier. In some cases where we required more detail or found the descriptions insufficient, we also consulted the web pages and the deliverables produced by the projects, but due to the high number of projects under consideration, this has been the exception rather than the rule. 


\section{ANALYSIS}

\section{A. ICT for Surveillance}

Within the policy documents, ICT is described as a tool to monitor different phenomena, to classify them, and to act upon those classifications. For example, the 2009 work programme states that " $[\mathrm{t}]$ he power grid needs new ICT-based monitoring and control systems to take on its growing complexity and distribution" [17, p.62]. In this statement, the value of ICT is in its capability to monitor complex exchanges as they happen. The same idea also appears in a different context in one of the objectives within the 2011 work programme:

Home energy controlling hubs that will collect realtime or near real-time data on energy consumption data from smart household appliances and enable intelligent automation. [18, p.75]

The role of the technologies remain similar in the home context. Both examples begin from traces of energy consumption. In the first case, ICTs control power grids by monitoring activities of different sources that produce or require energy, and intervene upon that process based on the categorisations they produce. In the second case, ICTs are used to monitor the energy consumption of inhabitants as they use different appliances, and to categorise the activities to coincide with different automation schemes.

The projects financed by the work programmes within Challenge 6 retain the same role for ICT. For example, the SmartHG project gathers "real-time data about energy usage from residential homes and exploit[s] such data for intelligent automation pursuing two main goals: minimizing energy usage and cost for each home" [19]. Similarly, the FIEMSER project aims to build "a monitoring and control system to optimize in near-real time the local generation-consumption matching" [20], and the Adapt4EE project "treat[s] occupants as the central reference point" and "analys[es] occupancy behaviour (presence and movement)" [21].

The term optimisation also appears within the analysed texts as a challenge that can be solved using ICT. In optimisation problems, an ongoing process considered sub-optimal is improved by monitoring and categorising. The policy documents request the optimisation of different activities, such as "generation-consumption matching" [17, p.66], the "dynamics of energy supply and demand" [18, p.74], and the "use of energy" [18, p.79]. Although the term is commonly used, a clear definition of what would be considered optimal in these contexts is not provided. The variables that should be considered for the cases of optimisation, and the evaluation criteria by which the optimisations would be measured are also not described. The texts focus on demand and consumption, hinting that the variables to be optimised are found at the consumer level. Statements such as "behavioural changes in the society at large" [17, p.67] point in the same direction as well. This statement on behavioural change is noteworthy, and we explore this issue further in the next section.

Moving from policy documents to the project descriptions, the term optimisation continues to occur frequently. Although in some cases the term remains ambiguous, for example in the case of the project description for IDEAS ("a neighbourhood energy management tool to optimise energy production and consumption" [22]), in other cases the projects provide different targets for optimisation which entail different forms of evaluation. For example, the SmartHG description states that it aims to "minimise the home energy bill and usage (local optimisation) with respect to a given price policy computed to attain global (grid level) optimisation" [19].

In more general terms, the policy documents suggest several optimisation processes which aim to shape consumers energy consumption using ICT-based surveillance. This basic model is then reflected by numerous projects, all of which propose to implement some variants depending on what type of surveillance is used and which mechanisms are considered to shape energy consumption.

\section{B. Changing User Behaviour}

In their roles as surveillance tools, ICTs are used in attempts to change user behaviour. In the policy documents, this change is described as raising user awareness, and aiding users in decision making. Under the ICT for Energy Efficiency objective from the 2009 work programme, the aim of the projects are described as producing "[i]ntuitive user interfaces that help end-users save energy while maintaining the desired comfort levels" [17, p.66]. The intuitiveness of interfaces are emphasised as important properties for saving energy, and discomfort is positioned as the opposing force that must be avoided. The possibility of creating discomfort by introducing new ICTs into the lives of the inhabitants is preempted by this statement. The same work programme provides a little more detail on what users might require in a different objective, Environmental Services and Climate Change: "Projects should be driven by the possibility for a range of users, including non ICT-skilled users, to plug-in their own use cases and get access to customised information and decision support." [17, p.67] These users, now positioned as information seekers, provide their needs to the ICTs and receive advice from them.

Although the above statements hint at an aim to change behaviour, the policy documents themselves rarely mention behaviours explicitly, except in the case of driving, where projects are expected to develop "[n]ew tools, systems and services supporting energy-efficient driving and driver behaviour adaptation" [18, p.80]. The intent to alter behaviour using ICT is much more apparent in the project descriptions. The ENERsip project provides a typical example: "The outcome of the adoption of ENERsip will allow setting new behavioural patters in the society and reduce overall intense economic dependence on energy" [23]. Similarly, the FIEMSER project proposes "solutions based on a rational consumption of energy, local generation and an increase in the consciousness of the building owners towards their energy consumption habits" [20]. Both projects also echo the aim set by the work programmes to build intuitive interfaces while maintaining the desired comfort levels using the same words as the programme. Some projects propose different mechanisms for pro- 
moting behavioural change such as financial incentives [24], mixed reality technologies [22], as well as gaming and social networking [25].

The project descriptions construct a chain of causality beginning from ICT-based surveillance, continuing with behavioural change, and concluding with energy savings or reduced $\mathrm{CO}_{2}$ emissions. The tension in this chain is most visible when we consider the set of decisions that must be made to construct the these technologies. For example, what is required to set new behavioural patterns? Which patterns would be preferred, and how would those who do not behave in accordance with the patterns react to it? Considerations along these lines are not present in the texts we have examined.

\section{Consequences}

The primary outcome that Challenge 6 aims at is using ICT to "assist in reshaping the demand side of our energydependent society, reducing energy consumption, and subsequently $\mathrm{CO}_{2}$ emissions" [18, p.74]. In addition to environmental goals, the work programmes mention different bureaucratic requirements in reference to international commitments, and economic goals such as maintaining competitiveness, reinforcing industrial and technological positions, and establishing an open energy market [17].

More specifically, the programmes refer to the need to aid communication and the transfer for information as goals that should be met by the projects. For example, the 2011 document describes part of its focus as the "[f]uture electricity distribution grids applying seamless communications systems to increase the connectivity, management, automation and coordination between suppliers (including renewable sources), consumers and networks" [18, p.74]. In this statement, ICT makes different contexts more amenable to management by connecting them. Consumers and suppliers of energy are linked by the flow of information, and the connection reshapes the demand side of society.

The projects financed under different objectives within the challenge begin with these goals and provide more specific solutions. The GreenCom project describes a smart grid that provides "[a] value based demand control based on individual consumer contracts with attractive tariffs, reward/penalty clauses and other elements will allow intelligent energy demand management and control" [26]. In line with the goals of the work programme, in this project ICT allows the consumers to enter into contracts where they can be rewarded or penalised, aiding the management of their demands on the supplier side.

Surveillance technologies allow the projects to begin from residential contexts and link them to energy goals as well. The SmartHG project states its expected outcome as: "gathering real-time data about energy usage from residential homes and exploiting such data for intelligent automation pursuing two main goals: minimising energy usage and cost for each home" [19]. In contrast to the diverse ICTs proposed for saving energy, the home itself is represented as a homogeneous space for energy consumption surveillance. The PEBBLE project extends the homogeneity further:

Through user-interfaces humans act as sensors communicating their thermal comfort preferences to the PEBBLE system, and in return the PEBBLE system returns information with the goal of enhancing energy-awareness of the users. The generality of the proposed methodology affords a universality that transcends regional, behavioral, environmental or other variations. [27]

In this statement, ICT is used to abstract away the irrelevant properties of the inhabitants, and to construct their involvement as a vehicle for voicing input. By discarding local contexts, it becomes possible for the system to transcend variations and to offer a universal solution.

Not all projects describe their users primarily as sensors. For example, the INERTIA project offers "fine grained control [...] while also protecting privacy and autonomy on the local level, fully respecting prosumers preferences and needs." [28]. The inhabitants are described using the term prosumers, which this project defines as consumers who produce energy using the proposed technologies, although there is also a recognition that the systems come into contact with users who have different needs and different preferences. These users are made into a coherent group in this brief statement by referring to how they are expected to interact with the system. At the same time, the designers recognise the potentially different needs and preferences of the users, although the specifics of that difference are not defined in the descriptions.

In general, the work programmes and the project summaries reveal very little concern about the heterogeneity of the stakeholders involved in their visions. Goals pertaining to aiding trust, safety, and inclusion, or preventing privacy breaches, marginalisation, and exclusion are not voiced by these texts. The underlying assumption regarding outcomes is that energy savings and the reduction of emissions are bound to occur if the users cooperate with ICTs and individually make decisions compatible with the continued functioning of these systems.

\section{DISCUSSION}

In the texts we have analysed, surveillance systems generate information by monitoring individuals and recording traces of their behaviour. These traces are then communicated back to the individuals in an attempt to change their behaviour. Depending on the goals of the project, the individuals are either provided financial incentives to convince them to consume energy differently, or they are taught to behave differently using feedback generated by surveillance systems. As we have stated previously, the assumption that behaviour can be altered by providing information presumes a theoretical stance that identifies the individual's outcome of deliberation as the cause, and the behaviour as the effect. The theory postulates that the individual can be informed using ICT, and the individual, who is presumed to be acting rationally by applying a cost-benefit analysis to decisions, is expected to change their behaviour after receiving information from the 
surveillance systems. We argue that there are two problems with assuming this theoretical stance when designing and developing ICTs for sustainability. First, the assumption that behaviour can be changed using information about energy consumption is questionable. Second, it neglects to address the negative societal effects of employing surveillance to influence behaviour while arguing for increased surveillance.

The assumption about behaviour change through consumption feedback is questionable primarily because it overemphasises individual choice while neglecting a whole range of other factors involved in shaping human behaviour. Information may be important in influencing how people behave, but there are a series of events between rendering information and behavioural change. First, the information must reach the user. This is a matter of both timing and presentation. The user must have the time required to perceive and interpret the information, and it must be presented in a way that allows the user to do so while in the midst of other ongoing events. In a home setting this accounts for high variation of activities, not all of which come with a large degree of spare time. Second, the user must possess the experience as well as the analytical expertise to interpret the information and to figure out how to act upon it. When targeting virtually everyone, ranging from children to elders, from formally uneducated to professionals and academics, from people that have spent their whole life in their current setting to people who just arrived from a life spent on the other side of the planet, the user population displays an extreme variation with regard to ways of interpreting and acting upon information. In addition to taking the time and possessing the expertise to make sense of the information, the user must also have an interest in doing so. Both the policy texts and many of the project descriptions from our analysis seem to assume that people have a general interest in changing their behaviour to save energy. Alternatively, the texts assume that financial or altruistic incentives can motivate people to take an interest. It is difficult to find empirical evidence in support of this assumption. Even if people are informed and motivated to adopt new behaviour, a number of other factors may work against change. Established practices related to behaviour do not only constrain what type of behaviour is possible, they also help shape motivations to behave in certain ways. Social norms play an important role in affecting behaviour, as do physical constraints. One popular example in the energy saving domain is the possibility of automatically delaying the start of the laundry machine until early mornings when there is a surplus of electricity in the network, when it is cheap, or when the $\mathrm{CO}_{2}$ emissions from electricity production are low. However, that might imply having to hang the wet laundry to dry in the morning instead of at night, which would conflict well-established practices of morning routines filled with activities like having breakfast, sending children to school, and commuting to work. In addition, the noise from the washing machine centrifuging during early morning hours is likely to interfere with the common practice of sleeping at night. Therefore, the assumption that users will change their behaviour as a result of information pertaining to energy consumption being made available through an "intuitive" user interface, without careful consideration of other factors that affect this process, can in general be considered flawed.

The definition of the term optimal in the materials we have examined reflects the viewpoints of their designers and stakeholders. To identify optimal resource consumption, surveillance systems are proposed for monitoring ongoing events, and for classifying the recorded traces. However, categorisations and classifications are not neutral; they encode the preferences of their designers, show what their designers have deemed should be visible, and hide what they have deemed not worth seeing. For example, a shortcoming of the financial incentives is made apparent through the emphasis on the proposed exchange of lowering energy consumption to obtain financial benefits. The exchange contains an internal contradiction: the saving of energy motivated by financial gain can only be an optimal behaviour if it generates more income per unit time than other methods of wealth generation such as salaries. For those with higher incomes, the time and attention spent on acquiring the best deal from the system is less likely to be higher than their current income per unit time. On the other hand, those with lower incomes, those who would benefit more financially from the financial incentives, are only able to participate if they invest time and acquire the technological competence required to operate the systems. For those who are able to learn to operate the system, interpret its results, and make the necessary changes, the system grants certain benefits such as lower energy costs. Marginalisation becomes visible at this level, where those who are not able to interpret the system become unable to enjoy its benefits, primarily expressed as a financial gain in the documents (e.g., they pay more because they do not know when it is cheapest to run the washing machine). Thus, the technologically and financially privileged can afford to ignore the system and disregard the disadvantages of lost profit while the underprivileged are marginalised further. By targeting the overlap between technological competence and positive financial gain, the idea to provide financial incentives to motivate behavioural change minimises the number of users likely to benefit from the system.

The focus on individual behaviour and choice by different EU projects is questionable on its own. Given the limitations of the model, the possibility of change using such frameworks seems small. However, the problem is not limited to model choice. After all, different disciplines use different methods and theories to understand the world, and when those methods do not yield results, they may be revised, or other methods can be used in their place. The problem with using these particular theories, of providing information to change behaviour, is that it is done with very little attention to possible negative social consequences. Large sums of European research funding in ICT design and development, generated largely through taxation [29], are expended on technologies that may not contribute with any positive effect for society, but also induce negative social effects, partially due to their uncritical use of surveillance. We argue that the lack of focus on possible 
negative social effects like marginalisation, exclusion, privacy breaches, and reduced trust raised by these quantifying methods, both at the policy level and at the project level, points to a general lack of engagement with these issues among ICT projects financed under FP7.

By opening the judgements of the designers to critical debate, concerns can be identified, voiced, discussed, and ultimately resolved through negotiation, preferably as an integral part of the design process. In this view, conflict is assumed to be a necessary component of social negotiation, but debates about assumptions can aid in minimising structural conflict that deeply advantages some subjects over others. If the assumptions are not challenged early in the design process, it becomes possible for the failure of the system to be blamed on the non-cooperative behaviour of the inhabitants. The system goes unused, and the end result does not contribute towards sustainability goals.

\section{CONCLUSion}

In summary, our analysis shows that the belief that human behaviour can be monitored at the individual level to generate different signals, and that these signals can be used to influence individuals to behave differently is well represented in both FP7 policy documents and descriptions of funded FP7 projects related to sustainability and energy efficiency. However, this is promoted and proposed almost entirely without references to the great number of other factors that determines human behaviour. Failing to do so, the texts provide little evidence to the fact that the potential behavioural change caused by this chain of events holds any promise for positively affecting energy consumption.

Meanwhile, both FP7 policy documents and descriptions of funded projects present a view of human behaviour and society seen primarily from a quantifying, managerial position. This position privileges counting, tracking, and efficiency, but it fails to address potential negative effects introduced by the surveillance technology, such as marginalisation, exclusion, and discrimination. In the policy documents and the project descriptions, surveillance systems are used to assist in reaching sustainability goals, and the potential positive effects are highlighted without considering the undesirable consequences of surveillance for those that are to be monitored.

This is not to suggest that there is no potential in applying surveillance technologies for the purpose of achieving sustainability goals with regard to environmental and energy efficiency. This may still very well be the case. However, in order to realise that, the design space must be expanded to cover more factors involved in shaping human behaviour, and the judgements of the designers and policy makers must be voiced in a critical debate where concerns for social effects can be identified, analysed, and discussed.

\section{REFERENCES}

[1] E. Shove, "Beyond the ABC: climate change policy and theories of social change," Environment and Planning. A, vol. 42, no. 6, pp. 12731285, 2010.
[2] - "Changing human behaviour and lifestyle: A challenge for sustainable consumption?" in Consumption - Perspectives from Ecological Economics, I. Røpke and L. Reisch, Eds. Elgar, 2005, pp. 111-132.

[3] P. Dourish, "HCI and environmental sustainability: The politics of design and the design of politics," in Proceedings of the 8th ACM Conference on Designing Interactive Systems, 2010, pp. 1-10.

[4] H. Brynjarsdóttir, M. Håkansson, J. Pierce, E. Baumer, C. DiSalvo, and P. Sengers, "Sustainably unpersuaded: How persuasion narrows our vision of sustainability," in Proceedings of the 2012 ACM Annual Conference on Human Factors in Computing Systems, 2012, pp. 947 956.

[5] J. Pierce, D. J. Schiano, and E. Paulos, "Home, habits, and energy: Examining domestic interactions and energy consumption," in Proceedings of the SIGCHI Conference on Human Factors in Computing Systems, 2010, pp. 1985-1994.

[6] Y. A. Strengers, "Designing eco-feedback systems for everyday life," in Proceedings of the SIGCHI Conference on Human Factors in Computing Systems, 2011, pp. 2135-2144.

[7] T. A. Rodden, J. E. Fischer, N. Pantidi, K. Bachour, and S. Moran, "At home with agents: Exploring attitudes towards future smart energy infrastructures," in Proceedings of the SIGCHI Conference on Human Factors in Computing Systems, 2013, pp. 1173-1182.

[8] T. Dillahunt, J. Mankoff, E. Paulos, and S. Fussell, "It's not all about green: Energy use in low-income communities," in Proceedings of the 11th International Conference on Ubiquitous Computing, 2009, pp. 255264.

[9] C. DiSalvo, P. Sengers, and H. Brynjarsdóttir, "Mapping the landscape of sustainable HCI," in Proceedings of the SIGCHI Conference on Human Factors in Computing Systems, 2010, pp. 1975-1984.

[10] K. Ball, D. Lyon, and K. Haggerty, Eds., Routledge Handbook of Surveillance Studies. Routledge, 2012.

[11] D. Lyon, Surveillance Studies: An Overview. Cambridge: Polity, 2007.

[12] O. H. J. Gandy, "Quixotics Unite! Engaging the Pragmatists on Rational Discrimination," in Theorizing Surveillance: The Panopticon and Beyond, D. Lyon, Ed. New York: Routledge, 2006, pp. 182-205.

[13] G. C. Bowker and S. L. Star, Sorting Things Out: Classification and Its Consequences. Cambridge, MA: The MIT Press, 2000.

[14] B. Cakici, "Sustainability through surveillance: ICT discourses in design documents," Surveillance \& Society, vol. 11, no. 1/2, pp. 177-189, 2013. [Online]. Available: http://library.queensu.ca/ojs/index. $\mathrm{php} /$ surveillance-and-society/article/view/sustainability

[15] EC, "European Commission. CORDIS: FP7," 2013. [Online]. Available: http://cordis.europa.eu/fp7/cooperation

[16] - "European Commission. CORDIS: Homepage," 2013. [Online]. Available: http://cordis.europa.eu/home_en.html

[17] — , "European Commission. Work Programme 2009, Cooperation Theme 3, ICT - Information and Communication Technologies," November 2008, c(2008)6827.

[18] —, "European Commission. Work Programme 2009, Cooperation Theme 3, ICT - Information and Communication Technologies," July 2010, c(2010)4900.

[19] SmartHG, "Projects : Search. CORDIS," 2012. [Online]. Available: http://cordis.europa.eu/projects/rcn/105541_en.html

[20] FIEMSER, "Projects : Search. CORDIS," 2013. [Online]. Available: http://cordis.europa.eu/projects/rcn/93803_en.html

[21] Adapt4EE, "Projects : Search. CORDIS," 2011. [Online]. Available: http://cordis.europa.eu/projects/rcn/100798 en.html

[22] IDEAS, "Projects : Search. CORDIS," 2012. [Online]. Available: http://cordis.europa.eu/projects/rcn/105738 en.html

[23] ENERsip, "Projects : Search. CORDIS," 2013. [Online]. Available: http://cordis.europa.eu/projects/rcn/93727_en.html

[24] E-Price, "Projects : Search. CORDIS," 2013. [Online]. Available: http://cordis.europa.eu/projects/rcn/93745_en.html

[25] EEPOS, "Projects : Search. CORDIS," 2012. [Online]. Available: http://cordis.europa.eu/projects/rcn/105854_en.html

[26] GreenCom, "Projects : Search. CORDIS," 2012. [Online]. Available: http://cordis.europa.eu/projects/rcn/105697_en.html

[27] PEBBLE, "Projects : Search. CORDIS," 2012. [Online]. Available: http://cordis.europa.eu/projects/rcn/93732_en.html

[28] INERTIA, "Projects : Search. CORDIS," 2012. [Online]. Available: http://cordis.europa.eu/projects/rcn/105543_en.html

[29] EC, "The Budget Explained - Where Does the Money Come From?" 2013. [Online]. Available: http://ec.europa.eu/budget/explained/budg system/financing/fin_en.cfm 